\title{
Use of $\alpha$-cyclodextrin to Promote Clean and Environmentally Friendly Disinfection of Phenolic Substrates via Chlorine Dioxide Treatment
}

\author{
Sauradip Chaudhuri ${ }^{1}$, Dana J. DiScenza ${ }^{2}$, Thomas B. Boving ${ }^{3}$, Alan Burke ${ }^{4}$ and \\ Mindy Levine ${ }^{5 *}$ \\ ${ }^{1}$ McGovern Medical School, University of Texas Health Science Center at Houston, Houston, TX, United States, \\ ${ }^{2}$ Department of Chemistry, University of Virginia, Charlottesville, VA, United States, ${ }^{3}$ Department of Geosciences/Department \\ of Civil and Environmental Engineering, University of Rhode Island, Kingston, RI, United States, ${ }^{4}$ Independent Researcher, \\ North Kingstown, RI, United States, ${ }^{5}$ Department of Chemical Sciences, Ariel University, Ariel, Israel
}

OPEN ACCESS

Edited by:

Yong Yao,

Nantong University, China

Reviewed by:

Yang Wang,

Nantong University, China

Chao-Guo Yan,

Yangzhou University, China

Ruibing Wang,

University of Macau, China

*Correspondence:

Mindy Levine

mindy.levine@gmail.com

Specialty section:

This article was submitted to

Supramolecular Chemistry,

a section of the journal

Frontiers in Chemistry

Received: 04 May 2020

Accepted: 22 June 2020

Published: 31 July 2020

Citation:

Chaudhuri S, DiScenza DJ, Boving TB, Burke $A$ and Levine $M$

(2020) Use of $\alpha$-cyclodextrin to

Promote Clean and Environmentally Friendly Disinfection of Phenolic Substrates via Chlorine Dioxide Treatment. Front. Chem. 8:641. doi: 10.3389/fchem.2020.00641
The use of chlorine dioxide to disinfect drinking water and ameliorate toxic components of wastewater has significant advantages in terms of providing safe water. Nonetheless, significant drawbacks toward such usage remain. These drawbacks include the fact that toxic byproducts of the disinfection agents are often formed, and the complete removal of such agents can be challenging. Reported herein is one approach to solving this problem: the use of $\alpha$-cyclodextrin to affect the product distribution in chlorine dioxide-mediated decomposition of organic pollutants. The presence of $\alpha$-cyclodextrin leads to markedly more oxidation and less aromatic chlorination, in a manner that is highly dependent on analyte structure and other reaction conditions. Mechanistic hypotheses are advanced to explain the cyclodextrin effect, and the potential for use of $\alpha$-cyclodextrin for practical wastewater treatment is also discussed.

Keywords: cyclodextrin, bisphenol (BPA), 2-phenylphenol, hydrophobic encapsulation, chlorination

\section{INTRODUCTION}

The decontamination of the water supply from a variety of organic pollutants (Cravotto et al., 2005), including phthalates (Przybylinska and Wyszkowski, 2016), biphenyls (Benoit et al., 2016), and bisphenol derivatives (Onundi et al., 2017) is an important challenge with a variety of industrial and public health applications (Foo and Hameed, 2010; Shah et al., 2016). Methods to achieve such decontamination to facilitate access to clean drinking water tend to rely on the application of large quantities of disinfectants, oxidants, or decomposition reagents (Kim et al., 1999), with newer methods including the use of photochemical (Laxma Reddy et al., 2017), electrochemical (Oturan et al., 2009), and sonochemical (Joseph et al., 2009) decontamination procedures. While these methods are effective in reducing the quantities of known organic pollutants (Besner et al., 2008), the decomposition products of both the pollutants and of chemicals used for disinfection have not been well-characterized (Wang et al., 2014), and methods to remove such decomposition products from the water stream are poorly developed. This is particularly concerning because many of these decomposition products are likely to have similar or even worse toxicities compared to their associated starting materials (Li and Mitch, 2018).

One popular disinfection agent is chlorine dioxide, which has been used for the decontamination of wastewater from pathogens (Banach et al., 2015), as part of the seawater desalination process (Kim et al., 2015), and for the removal of antibiotics and other pharmaceuticals from drinking 


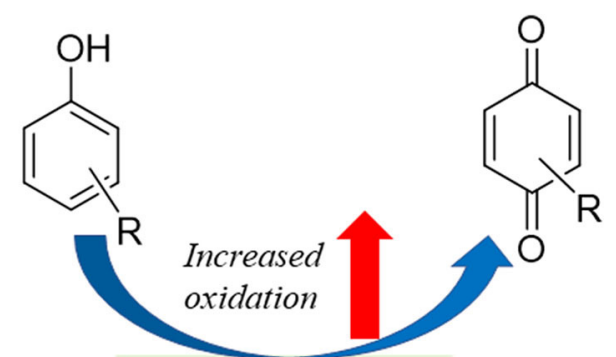

Chlorine dioxide $+\alpha-C D$

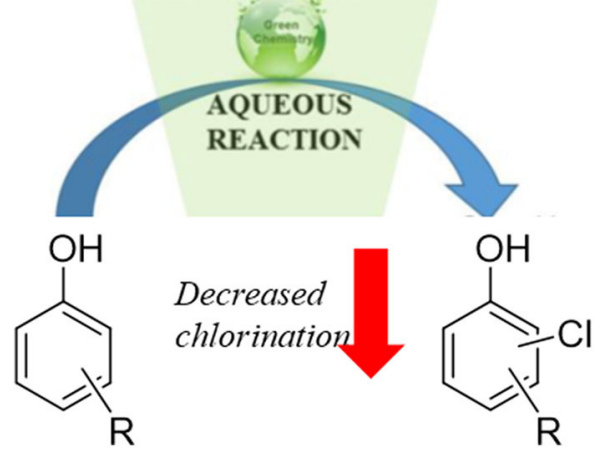

Graphical Abstract | Chlorine dioxide-mediated decontamination of aromatic pollutants in water in the presence of $\alpha$-cyclodextrin ( $\alpha$-CD) changes the reaction pathway, leading to decreased chlorination, and increased oxidation.

water (Dodd, 2012). Despite the widespread usage of chlorine dioxide, concerns remain about its toxicity (Ma et al., 2017), and about the toxicity of pollutant byproducts that result from chlorine dioxide treatment (Colman et al., 2011). Efforts to mitigate this toxicity have focused on alternative disinfection treatments (Meireles et al., 2016), on immobilization of chlorine dioxide to minimize the diffusion of toxic byproducts ( $\mathrm{He}$ et al., 2014), and on the combined use of chlorine dioxide and other water treatments (Hsu and Huang, 2015). The use of supramolecular constructs and/or adducts of chlorine dioxide as strategies for mitigating chlorine dioxide-induced water treatment toxicity has not been reported to date, despite the fact that chlorine dioxide is known to form a variety of supramolecular adducts (Loginova et al., 2011; Palcso et al., 2019), including with $\alpha$-cyclodextrin (Wambaugh et al., 2013). Moreover, supramolecular association with common organic pollutants is well-known, including cyclodextrin complexation with the classes of pollutants mentioned above [phthalates (Cromwell et al., 2019), biphenyls (Serio et al., 2013), and bisphenols (DiScenza et al., 2018)]. Finally, supramolecular complexation in general (Chang et al., 2017), and cyclodextrin complexation in particular (Aiassa et al., 2016), has been shown to result in significantly altered and often reduced toxicities, which provides another potential avenue by which toxicity of the water stream can be mitigated.

In general, cyclodextrin complexation has been shown to rely heavily on hydrophobic association of hydrophobic small molecules inside the hydrophobic interior cavity of the cyclodextrin hosts. Such host-guest complexes have a strong dependence on the steric complementarity between the cavity size and the size of the guest, with single aromatic ring compounds reported to bind strongly in $\alpha$-cyclodextrin (Connors and Pendergast, 1984; Pendergast and Connors, 1985) and larger aromatic (and hydrophobic aliphatic compounds) reported to bind in $\beta$-cyclodextrin (Celebioglu et al., 2019; Yu et al., 2019). Of note, moving to the even larger $\gamma$-cyclodextrin oftentimes results in the formation of ternary complexes, where two small molecule guests bind simultaneously inside the larger $\gamma$-cyclodextrin core (Hamai, 2010; Saokham et al., 2018). For the most common analytes involved in aqueous contamination (vide infra), the single aromatic rings of these analytes indicate that they are likely to bind strongly in the $\alpha$-cyclodextrin cavity. Such strong and sterically matched binding, in turn, is expected to affect the reactivity of these substrates and the distribution of products obtained, an expectation that was effectively borne out by the results of our experiments (vide infra). The use of larger cyclodextrins, by contrast, would lead to the formation of less sterically matched complexes, which would in turn impart lower selectivities and lower overall cyclodextrin-induced effects.

Recent reports from our research groups have focused on the design, optimization, and sensing applications of cyclodextrin complexes (for the Levine group) (Serio et al., 2015; Chaudhuri et al., 2018; Haynes et al., 2019), and on the engineering, deployment, and evaluation of water purification filters (for the Boving group) (Schifman et al., 2016; Eberle et al., 2017; Blanford et al., 2018), which combined have provided us with unique insight into the potential of cyclodextrins to benefit the water purification process. Reported herein are the results of our investigations into the effect of $\alpha$-cyclodextrin complexation on chlorine dioxide-based water treatment, and how such complexation affects the quantity and distribution of degradation byproducts. Mechanistic insight into the specific role of $\alpha$ cyclodextrin is also discussed.

\section{EXPERIMENTAL SECTION}

\section{Materials and Methods}

${ }^{1} \mathrm{H}$ NMR experiments were conducted using a $400 \mathrm{MHz}$ Bruker Avance spectrometer with $\mathrm{D}_{2} \mathrm{O}$ as a solvent. GC-MS analyses of reaction mixtures were carried out using a Shimadzu GCMSQP2020 instrument. All chemicals were purchased from Sigma Aldrich chemical company or from Fisher Scientific and were used as received, without further purification.

\section{Method for the Preparation of Chlorine Dioxide Solution}

An aqueous chlorine dioxide suspension was generated from the treatment of a solution of $\mathrm{NaClO}_{2}$ (ERCOPure $\left.{ }^{\mathrm{TM}} 7.5\right)$ with activated $\mathrm{HCl}$. A typical generation procedure involved the addition of $4 \mathrm{~mL}$ of $30-36 \% \mathrm{HCl}$ to a mixture of $17.5 \mathrm{~mL}$ of $\mathrm{ADOX}^{\mathrm{TM}} 7.5$ and $200 \mathrm{~mL}$ of deionized water. The reaction mixture was kept in a dark Amber bottle for $\sim 24 \mathrm{~h}$ at room temperature. Prior to usage, the reaction mixture was further diluted to render a final chlorine dioxide concentration of $\sim 1,095$ ppm (confirmed via hand-held colorimetry using a Hach Digital Titrator). 

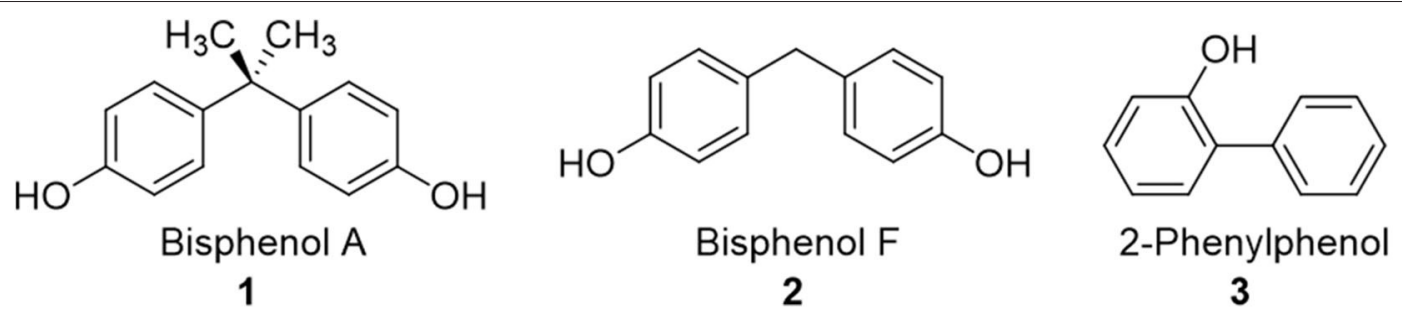

FIGURE 1 | Structures of common aqueous pollutants investigated herein as substrates for chlorine dioxide-mediated degradation.

\section{General Method for the Complexation of Chlorine Dioxide With $\alpha$-cyclodextrin}

The complexation of chlorine dioxide with $\alpha$-cyclodextrin was obtained by mixing a solution of $6.2 \mathrm{~mL}$ of $1,100 \mathrm{ppm}$ of $\mathrm{ClO}_{2}$ $(100 \mu \mathrm{mol})$ with solid $\alpha$-cyclodextrin $(584 \mathrm{mg} ; 600 \mu \mathrm{mol})$ for 15-20 min.

\section{General Method for Determining the Reaction Progression}

The reaction mixture containing the organic analyte (compounds 1-3, Figure 1) was treated with $\mathrm{ClO}_{2}$ and allowed to react at the specified temperature for a certain amount of time, after which time the mixture was treated with concentrated sodium sulfite $\left(\mathrm{Na}_{2} \mathrm{SO}_{3}\right)$ solution to quench the excess chlorine dioxide. The resulting solution was extracted with ethyl acetate. An aliquot of the organic phase was injected into the GC-MS for analysis, which enabled us to identify unreacted starting material, as well as new peaks corresponding to the formation of a variety of oxidation and chlorination products.

\section{General Method for Measuring the Binding of Analytes in $\alpha$-cyclodextrin}

Binding of analytes with $\alpha$-cyclodextrin was investigated via ${ }^{1} \mathrm{H}$ NMR titrations (Roselet and Kumari, 2017). A mixture of analytes $(20 \mu \mathrm{mol})$ with $\alpha$-cyclodextrin (0.0-5.0 equivalents) in $\mathrm{D}_{2} \mathrm{O}$ were investigated via ${ }^{1} \mathrm{H}$ NMR spectroscopy, and the resulting shifts in the positions of the NMR signals were used to confirm supramolecular complexation.

\section{RESULTS AND DISCUSSION}

\section{Analyte Selection}

There are a broad variety of organic pollutants that contaminate water supplies, including phthalates, biphenyls, and bisphenol derivatives (vide supra). We have selected three common pollutants to focus on in this paper, all of which have been reported to interact with $\alpha$-cyclodextrin: bisphenol A (BPA) (analyte 1) (Araki et al., 2001), bisphenol F (BPF) (analyte 2) (Xiao et al., 2007), and 2-phenylphenol (analyte 3) (Burkert et al., 1981), with the expectation that supramolecular interactions of the pollutants with $\alpha$-cyclodextrin is likely to affect their chlorine dioxide-mediated degradation. Moreover, the selection of three pollutants with similar structures is expected to provide important insight into the structural selectivity of $\alpha$-cyclodextrin complexation, and how such selectivity affects the chlorine dioxide-mediated degradation processes. Finally, the inclusion of $\mathrm{BPF}$ in addition to BPA is important, as BPA derivatives such as BPF are increasingly used as commercially available substitutes for BPA (Bjornsdotter et al., 2017; Wu et al., 2018), with evidence indicating analogous or even worse toxicity compared to BPA (den Braver-Sewradj et al., 2020).

\section{Initial Screening}

Initial screening of reaction conditions started with room temperature treatment of analyte $\mathbf{1}$ with chlorine dioxide and $\alpha$-cyclodextrin (0-60 equivalents relative to the substrate). BPA was found to undergo decomposition into single aromatic ring oxidized units (quinols and quinones), as well as undergo chlorination on residual starting material to form chlorinated BPA analogs. Under these conditions, increasing the equivalents of cyclodextrin led to a dramatic increase in the ratio of oxidation products to chlorination products, from a ratio of 3.2 without any cyclodextrin to a ratio of oxidation to chlorination of 49 measured at the highest concentration of $\alpha$-cyclodextrin (Figure 2).

However, even after $24 \mathrm{~h}$, significant unreacted starting material remained (up to $72 \%$ ). Raising the reaction temperature slightly, to $40^{\circ} \mathrm{C}$, resulted in complete consumption of the starting material but dramatic changes in the reaction products, with nearly exclusive formation of chlorinated oxidation products chloroquinol and chloroquinone (Figure 3). This significant change in consumption of the starting material with only a mild increase in the temperature of the reaction is likely due to increased reaction kinetics at the elevated temperature. The stability of the cyclodextrin complexes is likely also affected by the increased reaction temperature, which in turn leads to changes in the distribution of products observed. Increasing the equivalents of $\alpha$-cyclodextrin in this system led to moderate increases in the ratio of chloroquinol to chloroquinone obtained, with overall limited changes in the overall oxidation products obtained (Table 1). This could likely be due to the stronger binding of quinols to $\alpha$-cyclodextrin, thereby inhibiting their subsequent oxidation into quinones.

In contrast to the results obtained for analyte 1 , treatment of bisphenol $\mathrm{F}$ (analyte 2) with chlorine dioxide at $40^{\circ} \mathrm{C}$ led to $100 \%$ oxidation products, with both unsubstituted quinols and chloroquinols formed (Figure 4). The ratio of oxidation products ( $100 \%$ of the product mixture) to chlorination products (namely, the formation of chloroquinols) was calculated, and the results 

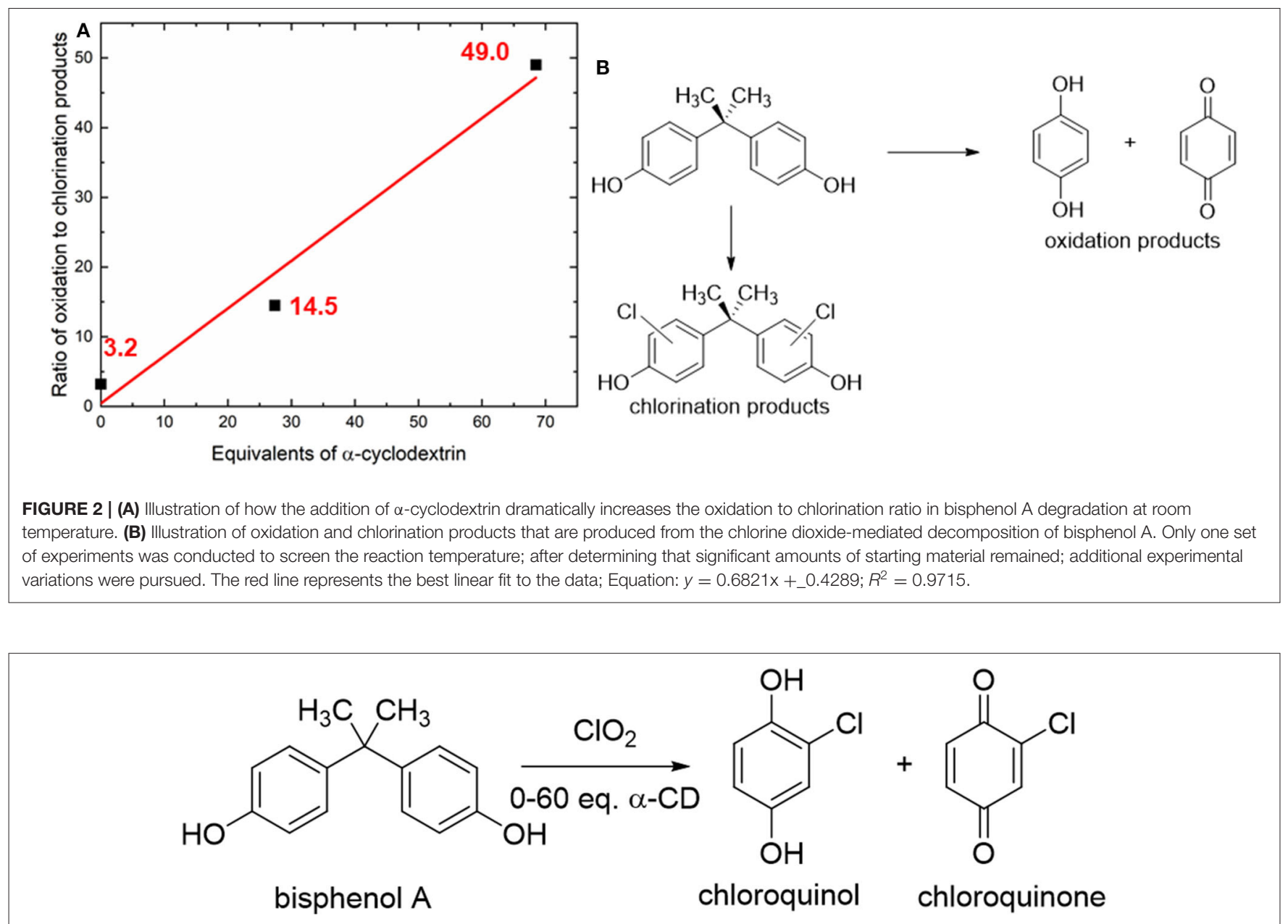

FIGURE 3 | Illustration of the degradation reaction of bisphenol A upon treatment with chlorine dioxide at $40^{\circ} \mathrm{C}$.

TABLE 1 | Effects of $\alpha$-cyclodextrin addition on the distribution of decomposition products obtained from chlorine dioxide treatment of bisphenol $\mathrm{A}^{\mathrm{a}}$.

\begin{tabular}{lcc}
\hline $\begin{array}{c}\text { Equivalents of } \\
\alpha \text {-cyclodextrin }\end{array}$ & $\begin{array}{r}\text { Ratio of chloroquinol } \\
\text { to chloroquinone }\end{array}$ & Overall oxidation product \% \\
\hline 0 & 1.9 & $56.2 \%$ \\
27.4 & 3.5 & $53.6 \%$ \\
68.5 & 4.3 & $55.3 \%$
\end{tabular}

${ }^{a}$ Reactions were run at $40^{\circ} \mathrm{C}$ for $24 \mathrm{~h}$.

summarized in Table 2. Of note, substantial increases in the ratio of quinol to chloroquinol with increasing concentration of cyclodextrin indicates a decrease in the chlorination byproducts, which strongly suggests that cyclodextrin complexation plays a role in inhibiting that reaction pathway (vide infra).

In contrast to analytes $\mathbf{1}$ and $\mathbf{2}$, analyte $\mathbf{3}$ (2-phenylphenol) underwent complete decomposition with $\mathrm{ClO}_{2}$ treatment, yielding a much more complex product profile. Unlike analytes $\mathbf{1}$ and 2, which formed predominantly single aromatic ring oxidation products, the chlorine dioxide treatment of analyte
3 led to only minor amounts of such products, with the majority of oxidation products maintaining the core biphenyl structure. Such differences in product distribution between the analytes strongly suggests that the bridging methylene unit of the bisphenol structures of $\mathbf{1}$ and $\mathbf{2}$ (absent in analyte 3 ) provided a site for $\mathrm{C}-\mathrm{C}$ bond cleavage that enabled single aromatic ring products to form. In addition to the biphenyl-containing oxidation products, a variety of chlorinated products were also formed, most of which resulted from chlorination of the initially formed oxidized compounds (Figure 5). The ratio of oxidation products to chlorination products formed from chlorine dioxidemediated decomposition of analyte 3 , with increasing equivalents of $\alpha$-cyclodextrin effectively protecting the aromatic ring from undesired chlorination reactions (as shown by increasing values of the oxidation to chlorination ratio observed, Table 2 ).

Overall, the results obtained for analyte $\mathbf{1}$ at room temperature, and analytes 2 and 3 at $40^{\circ} \mathrm{C}$ indicate that the presence of cyclodextrin markedly increases the percentage of non-chlorinated oxidation products formed, with the one anomalous result, obtained for the chlorine dioxide treatment of analyte 1 at $40^{\circ} \mathrm{C}$, discussed later in the manuscript. Of note, 


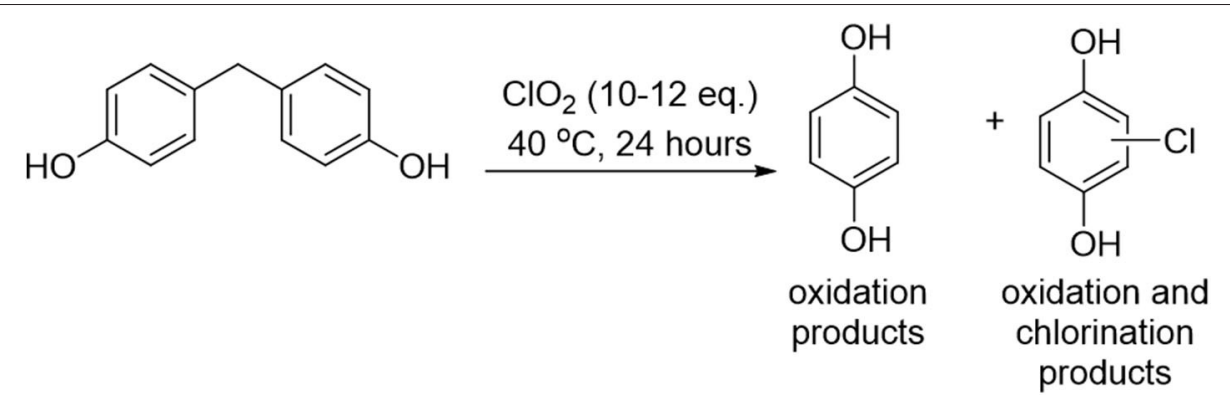

FIGURE 4 | Illustration of how the chlorine dioxide-mediated decomposition of bisphenol F leads to the formation of quinols and chloroquinols exclusively.

TABLE 2 | Ratio of oxidation to chlorination products formed by treating aromatic analytes $\mathbf{2}$ and $\mathbf{3}$ with chlorine dioxide with varying cyclodextrin equivalents ${ }^{\mathrm{a}}$.

\begin{tabular}{lccc}
\hline Analyte & $\mathbf{0}$ eq. $\boldsymbol{\alpha - C D}$ & $\mathbf{2 4}$ eq. $\boldsymbol{\alpha - C D}$ & $\mathbf{6 0}$ eq. $\boldsymbol{\alpha - C D}$ \\
\hline $\mathbf{3}$ & 1.2 & 1.5 & 1.5 \\
\hline
\end{tabular}

${ }^{a}$ Reactions were run at $40^{\circ} \mathrm{C}$ for $24 \mathrm{~h}$, and the ratio of oxidation to chlorination products was determined via GC-MS analysis.

both substrates with bridging methylene units (compounds 1 and 2) decomposed primarily into single aromatic ring oxidized units [(chloro)quinols and (chloro)quinones]. Such results have substantial relevance from a practical as well as a fundamental scientific perspective. From a practical perspective, chlorinated byproducts formed from chlorine dioxide mediated decomposition generally have higher reported toxicities than the non-chlorinated, oxidation products formed ( $\mathrm{Li}$ and Mitch, 2018). As a result, the ability to decrease the relative amount of chlorinated products through $\alpha$-cyclodextrin addition is particularly attractive, especially as $\alpha$-cyclodextrin itself has almost no reported toxicity (Cal and Centkowska, 2008), and in fact has been used for a variety of biomedical applications due to its generally recognized safety (Szente et al., 2018). From a fundamental perspective, the fact that $\alpha$-cyclodextrin complexation suppresses the chlorination processes is likely due to hydrophobic encapsulation of the phenyl rings in the cyclodextrin cavity, in a way that provides steric shielding and prevents aromatic chlorination from occurring (vide infra). Such supramolecular shielding provides insight into the mechanism of cyclodextrin complexation, how such complexation depends on the structure of the encapsulated guest, and how such complexes affect guest reactivity.

\section{Mechanistic Investigations}

There are multiple fundamental mechanistic questions involved in this process, including how $\alpha$-cyclodextrin affects the product distribution of chlorine dioxide mediated degradation, as well as how variations in substrate structure affect product distribution and the underlying reaction mechanism. Most likely, the binding of phenyl groups in the cyclodextrin cavity (Figure 6) provides supramolecular steric shielding from undesired aromatic chlorination. Moreover, the benzylic position on the substituent, which must be accessed to effect oxidation reactions, remains relatively unhindered. Electronic activation of that benzylic position through hydrogen bonding to the rims of cyclodextrins can also accelerate the desired oxidation reactions, and evidence for such activation is provided through ${ }^{1} \mathrm{H}$ NMR analysis (vide infra).

Overall, the treatment of the analytes with chlorine dioxide led to highly analyte-specific results. Both analytes $\mathbf{1}$ and $\mathbf{2}$ yielded oxidation products with primarily single ring aromatic groups, whereas analyte $\mathbf{3}$ maintained its biphenyl structure, indicating that the bridging methylene group of analytes $\mathbf{1}$ and $\mathbf{2}$ plays a key role in facilitating oxidative bond cleavage. Differences in the product distribution of analytes $\mathbf{1}$ and $\mathbf{2}$ indicate a markedly more complex product mixture for analyte 1's treatment with chlorine dioxide, with major products of chloroquinol and chloroquinone and numerous minor products, generally with higher molecular weights (indicating radical-radical recombination). For analyte $\mathbf{1}$, the fact that the ratio of chloroquinol to chloroquinone increased with increasing equivalents of $\alpha$-cyclodextrin indicates that the cyclodextrin effectively inhibits oxidation of chloroquinol. In contrast, increasing the equivalents of $\alpha$-cyclodextrin in the analyte 2 decomposition process led to an increased ratio of quinol to chloroquinol, which indicates that the chlorination reaction pathway was inhibited by $\alpha$-cyclodextrin.

The proposed mechanism is further supported by ${ }^{1} \mathrm{H}$ NMR chemical shift studies of the analytes in presence of increasing equivalents of $\alpha$-cyclodextrin, and key results are summarized in Table 3 and Figure 7. In particular, all aromatic protons of the analytes demonstrated significant chemical shifts upon the addition of increasing concentrations of $\alpha$-cyclodextrin, supporting supramolecular encapsulation of the type shown in Figure 6. Moreover, for analytes $\mathbf{1}$ and 2, significant changes in chemical shift were also observed for the methyl group protons at the bridging carbon (for analyte 1) or for the protons directly on the methylene bridge (for analyte 2), which indicates the existence of significant non-covalent interactions between this part of the molecule and the cyclodextrin host (Yang et al., 2008). Such interactions are likely intermolecular hydrogen bonding between the hydroxyls located at the cyclodextrin rim and the protons between the aromatic rings, which in turn activates the benzylic position for the desired oxidation reactions. 


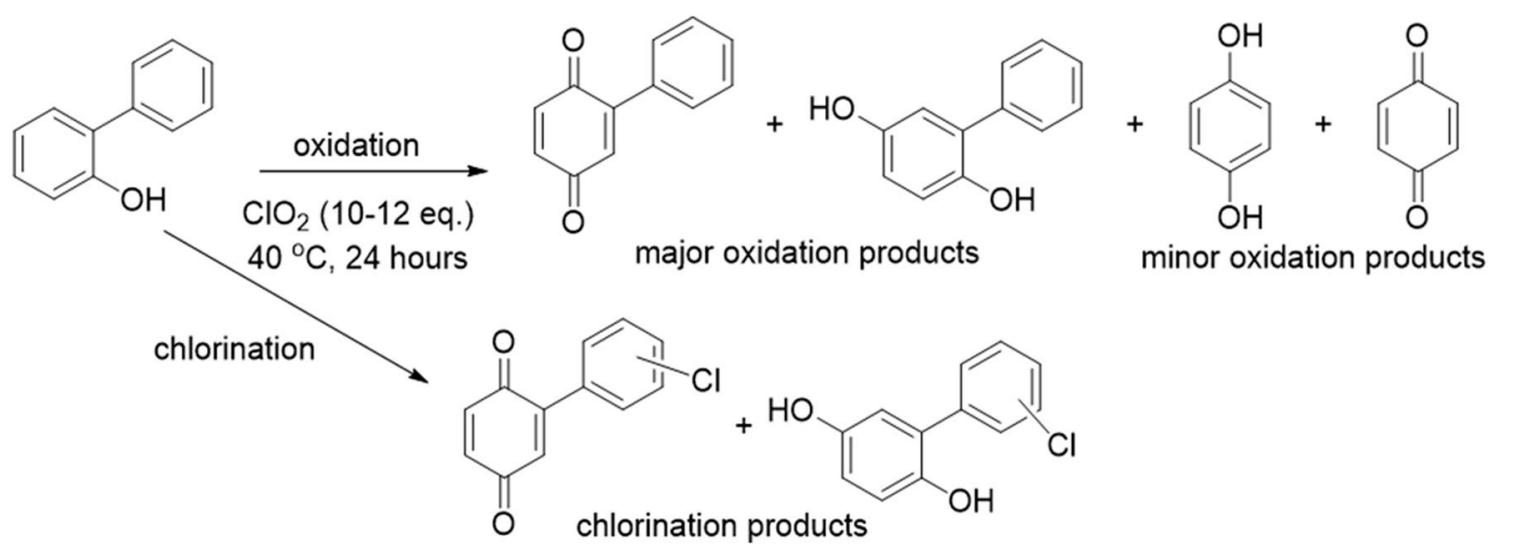

FIGURE 5 | Illustration of the products formed from chlorine dioxide-mediated decomposition of analyte 3.

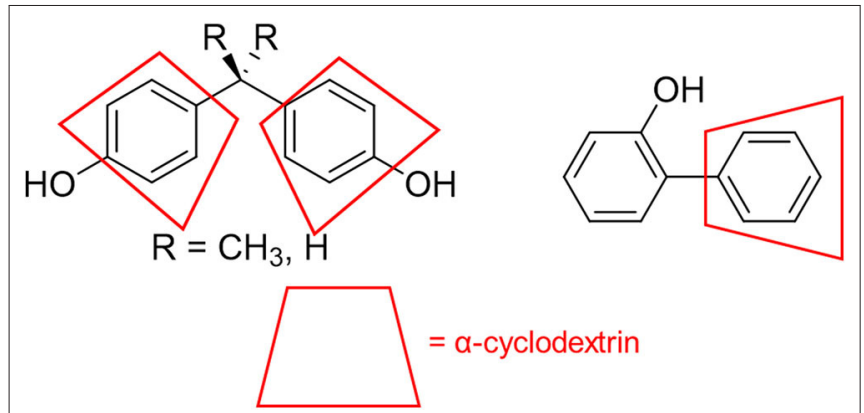

FIGURE 6 | Schematic illustration of how $\alpha$-cyclodextrin binds to aromatic rings on the pollutant structures to limit aromatic chlorination.

Of note, binding of aromatic compounds in cyclodextrin that leads to activation of benzylic positions through association with the cyclodextrin rim is a phenomenon that has been reported previously in the literature, both by our group (Chaudhuri et al., 2016) and by others (Andres and de Rossi, 2003; Lopez et al., 2007). In particular, a previous report by our group uses binding of aromatic rings in the cyclodextrin cavity to activate the benzylic position of benzylic alcohols and achieve effective and mild oxidation to the corresponding aldehydes (den BraverSewradj et al., 2020). Similarly, complexation in $\alpha$-cyclodextrin, reported herein, has the dual function of protecting the aromatic ring from undesired chlorination and of facilitating effective oxidation at the benzylic site.

\section{Practical Applications}

Real-world municipal waste water effluent samples, prior to chlorination, were used to simulate this mediated oxidation in practice. Such samples were doped with $100 \mathrm{mg} / \mathrm{L}$ of BPA (analyte 1), and then treated with chlorine dioxide in the presence or absence of $\alpha$-cyclodextrin. Results of these studies showed that $\alpha$-cyclodextrin promoted the decomposition of $\mathrm{BPA}$ to form hydroquinone and chlorohydroquinone, with
TABLE 3 | Changes in the ${ }^{1} \mathrm{H}$ NMR spectral signals of protons on bisphenol $\mathrm{A}$ as a function of added equivalents of $\alpha$-cyclodextrin $(\alpha-C D)^{a}$.

\begin{tabular}{lccc}
\hline Eq. of $\alpha-C D$ & $\begin{array}{c}\text { Methyl protons } \\
(\boldsymbol{\Delta} \mathbf{p p m})\end{array}$ & $\begin{array}{c}\text { Ortho protons } \\
(\boldsymbol{\Delta} \mathbf{p p m})^{\mathbf{b}}\end{array}$ & $\begin{array}{c}\text { Meta protons } \\
(\boldsymbol{\Delta} \mathbf{p p m})^{\mathbf{c}}\end{array}$ \\
\hline 0.5 & 0.0291 & 0.0044 & 0.0609 \\
1.0 & 0.0400 & 0.0071 & 0.0801 \\
1.5 & 0.0443 & 0.0077 & 0.0870 \\
2.0 & 0.0493 & 0.0089 & 0.0920 \\
3.0 & 0.0522 & 0.0088 & 0.0953 \\
5.0 & 0.0609 & 0.0135 & 0.1015
\end{tabular}

${ }^{a} \Delta p p m$ is defined as the difference in chemical shifts in the presence of cyclodextrin compared to the chemical shifts in the absence of cyclodextrin, according to the following equation.

$\Delta p p m=\delta_{\text {complex }}$ (chemical shifts in presence of $\left.\alpha-C D\right)-\delta_{\text {control }}$ (chemical shifts without $\alpha-C D)$.

${ }^{b}$ Ortho protons are defined as the protons that are at the ortho positions of the aromatic ring relative to the non-aromatic bridge.

${ }^{c}$ Meta protons are defined as the protons that are at the meta positions of the aromatic ring relative to the non-aromatic bridge.

substantially more of these products formed in the presence of $\alpha$ cyclodextrin compared to the decomposition run in the absence of cyclodextrin (Table 4). Nonetheless, the ratio of hydroquinone to chlorohydroquinone remained roughly unchanged by the addition of $\alpha$-cyclodextrin, a result which is surprising based on the documented ability of $\alpha$-cyclodextrin to affect this product distribution (vide supra). Reasons for this anomalous behavior may relate to the presence of interfering species in the wastewater sample. In particular, higher ionic strength and/or other species complexing with cyclodextrin sites can limit the ability to target specific compounds for specific oxidation mechanisms. Such treatment might be better suited in the long-term for industrial wastewater streams where a more consistent water matrix and a higher concentration of the targeted pollutant is available for cyclodextrin-mediated oxidation. We expect that additional modifications to our cyclodextrin-based system will allow for improved performance in such samples, possibly through combining the $\alpha$-cyclodextrin with other additives that will address the more complex nature of real-world aqueous samples. 


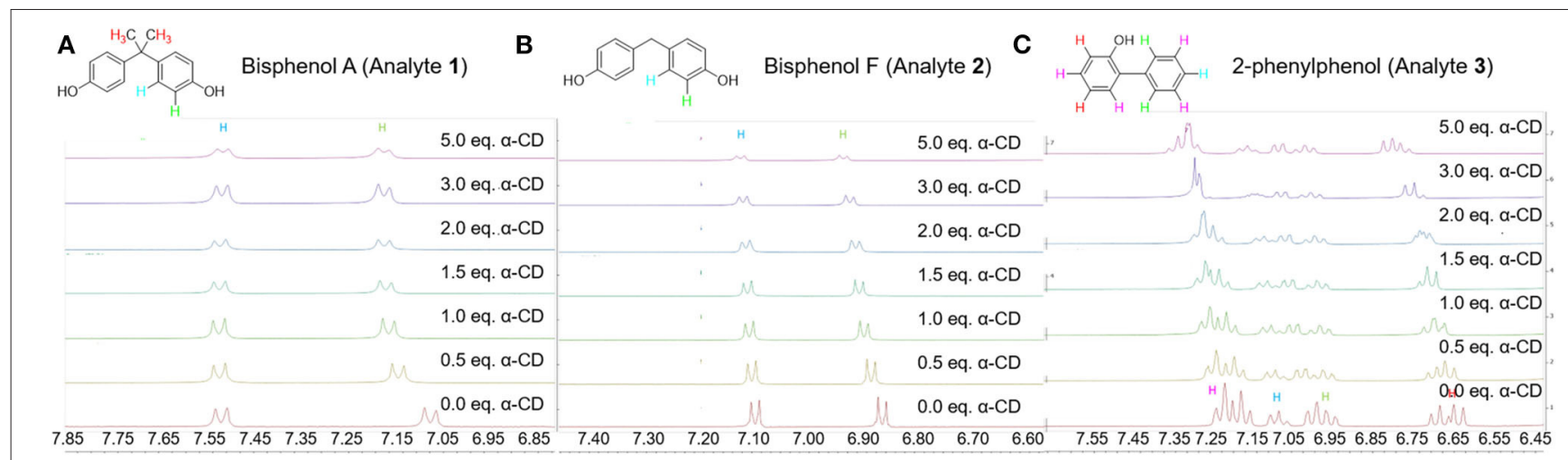

FIGURE 7 | Copies of ${ }^{1} \mathrm{H}$ NMR spectra of the aromatic protons of (A) analyte $\mathbf{1}$; (B) analyte $\mathbf{2}$; and (C) analyte $\mathbf{3}$ in the presence of increasing equivalents of $\alpha$-cyclodextrin.

TABLE 4 | Summary of the formation of hydroquinone and chlorohydroquinone from chlorine dioxide mediated decomposition of BPA in real-world wastewater samples, measured as normalized integrated peak emissions from GC-MS.

\begin{tabular}{lcc}
\hline Decomposition product & Without $\alpha-C D$ & With $\alpha-C D$ \\
\hline Hydroquinone $^{\mathrm{a}}$ & 0.17 & 0.31 \\
Chlorohydroquinone $^{\mathrm{a}}$ & 0.51 & 1.00 \\
Ratio of hydroquinone to chlorohydroquinone $^{\mathrm{b}}$ & 0.33 & 0.31 \\
\hline
\end{tabular}

a Values reported herein represent the integrated area of the GC-MS peaks that correspond to each analyte, with the results normalized so that the highest value peak (chlorohydroquinone in the presence of $\alpha-C D$ ) is equal to 1.0.

${ }^{b}$ Ratio of hydroquinone to chlorohydroquinone is calculated as the quotient of the integrated area of the peak corresponding to hydroquinone divided by the integrated area of the peak corresponding to chlorohydroquinone.

\section{CONCLUSIONS}

The ability to change the product distribution of chlorine dioxide mediated degradation of organic pollutants via supramolecular complexation of the pollutants has substantial practical benefit in improving wastewater treatment methodologies, and is of interest from a fundamental scientific perspective as well. Results reported herein highlight that the use of $\alpha$-cyclodextrin to bind small aromatic pollutants affects the accessibility of the structure to the chlorine dioxide reagent and the resulting distribution of oxidation to chlorination products in a way that is highly dependent on the structure of the reagent, the temperature of the treatment, and the molar equivalents of $\alpha$-cyclodextrin added, with most cases resulting in a marked decrease in the relative amounts of chlorinated byproducts obtained. Reasons for these effects rely on the supramolecular complexation of the pollutants, confirmed by ${ }^{1} \mathrm{H}$ NMR analysis, which cause steric shielding of the phenyl groups of the pollutants to undesired chlorination. Overall, the addition of $\alpha$-cyclodextrin generally increases the ratio of water-soluble oxidation products and decreases the amount of toxic chlorination products, through the addition of a non-toxic, sugar-based cyclodextrin additive. The results reported herein provide significant groundwork for further development of novel and highly effective water treatment procedures, and open the possibility of using cyclodextrinmediated complexation in other water treatment and pollutant removal processes.

\section{DATA AVAILABILITY STATEMENT}

All datasets generated for this study are included in the article/Supplementary Material.

\section{AUTHOR CONTRIBUTIONS}

SC ran the initial experiments on purified laboratory systems, identified system components, did $1 \mathrm{H}$ NMR analysis, and drafted the initial manuscript. DD ran experiments on realworld wastewater samples, finalized experiments that were left from SC, and edited the manuscript. TB provided intellectual insight throughout the project and edited the manuscript. $A B$ provided industrial perspective on the project, as well as realworld wastewater samples, and also edited the manuscript. ML provided intellectual insight on the project, worked with SC to draft the initial manuscript, and took responsibility for all edits and corrections to arrive at this final version. All authors contributed to the article and approved the submitted version.

\section{FUNDING}

International Dioxcide funded this work through a cooperative agreement with the University of Rhode Island. Fees to publish this work will be partially offset by an Open Access Fund at the University of Rhode Island and by discretionary funds at Ariel University.

\section{SUPPLEMENTARY MATERIAL}

The Supplementary Material for this article can be found online at: https://www.frontiersin.org/articles/10.3389/fchem. 2020.00641/full\#supplementary-material 


\section{REFERENCES}

Aiassa, V., Zoppi, A., Becerra, M. C., Albesa, I., and Longhi, M. R. (2016). Enhanced inhibition of bacterial biofilm formation and reduced leukocyte toxicity by chloramphenicol: $\beta$-cyclodextrin: $\mathrm{N}$-acetylcysteine complex. Carbohydr. Polym. 152, 672-678. doi: 10.1016/j.carbpol.2016.07.013

Andres, G. O., and de Rossi, R. H. (2003). Mechanism of phthalate ester hydrolysis in water and in cyclodextrin mediated reactions. ARKIVOC 127-138. doi: 10.3998/ark.5550190.0004.a14

Araki, M., Kawasaki, N., Nakamura, T., and Tanada, S. (2001). Removal of bisphenol A in soil by cyclodextrin derivatives. Toxicol. Environ. Chem. 79, 23-29. doi: 10.1080/02772240109358973

Banach, J. L., Sampers, I., Van Haute, S., and van der Fels-Klerx, H. J. (2015). Effect of disinfectants on preventing the cross-contamination of pathogens in fresh produce washing water. Int. J. Environ. Res. Public Health 12, 8658-8677. doi: 10.3390/ijerph120808658

Benoit, N., Dove, A., Burniston, D., and Boyd, D. (2016). Tracking PCB contamination in Ontario Great Lakes Tributaries: development of methodologies and lessons learned for watershed based investigations. J. Environ. Protect. 7, 390-409. doi: 10.4236/jep.2016.73035

Besner, M.-C., Servais, P., and Prevost, M. (2008). Efficacy of disinfectant residual on microbial intrusion: a review of experiments. J. Am. Water Works Assoc. 100, 116-130. doi: 10.1002/j.1551-8833.2008.tb09752.x

Bjornsdotter, M. K., de Boer, J., and Ballesteros-Gomez, A. (2017). Bisphenol A and replacements in thermal paper: a review. Chemosphere 182, 691-706. doi: 10.1016/j.chemosphere.2017.05.070

Blanford, W. J., Pecoraro, M. P., Heinrichs, R., and Boving, T. B. (2018). Enhanced reductive de-chlorination of a solvent contaminated aquifer through addition and apparent fermentation of cyclodextrin. J. Contaminant Hydrol. 208, 68-78. doi: 10.1016/j.jconhyd.2017.10.006

Burkert, W. G., Owensby, C. N., and Hinze, W. L. (1981). The use of an $\alpha$ cyclodextrin mobile phase in the thin-layer chromatographic separation of ortho, meta, and para substituted phenols. J. Liq. Chromatogr. 4, 1065-1085. doi: 10.1080/01483918108059604

Cal, K., and Centkowska, K. (2008). Use of cyclodextrins in topical formulations: practical aspects. Eur. J. Pharm. Biopharm. 68, 467-478. doi: 10.1016/j.ejpb.2007.08.002

Celebioglu, A., Topuz, F., Yildiz, Z. I., and Uyar, T. (2019). Efficient removal of polycyclic aromatic hydrocarbons and heavy metals from water by electrospun nanofibrous polycyclodextrin membranes. ACS Omega 4, 7850-7860. doi: 10.1021/acsomega.9b00279

Chang, Y.-X., Zhang, X.-M., Duan, X.-C., Liu, F., and Du, L.-M. (2017). Supramolecular interaction of methotrexate with cucurbit[7]uril and analytical application. Spectrochim. Acta A 183, 131-137. doi: 10.1016/j.saa.2017. 04.060

Chaudhuri, S., Verderame, M., Mako, T. L., Bandara, Y. M. N. D. Y., Fernando, A. I., and Levine, M. (2018). Synthetic $\beta$-cyclodextrin dimers for squaraine binding: effect of host architecture on photophysical properties, aggregate formation and chemical reactivity. Eur. J. Org. Chem. 2018, 1964-1974. doi: $10.1002 /$ ejoc.201800283

Chaudhuri, S., Zaki, H., and Levine, M. (2016). Environmentally friendly procedure for the aqueous oxidation of benzyl alcohols to aldehydes with dibromodimethylhydantoin (DBDMH) and cyclodextrin: scope and mechanistic insights. Synth. Commun. 46, 636-644. doi: 10.1080/00397911.2016.1161801

Colman, J., Rice, G. E., Wright, J. M., Hunter, E. S. III; Teuschler, L. K., Lipscomb, J. C., Hertzberg, R. C., et al. (2011). Identification of developmentally toxic drinking water disinfection byproducts and evaluation of data relevant to mode of action. Toxicol. Appl. Pharmacol. 254, 100-126. doi: 10.1016/j.taap.2011.02.002

Connors, K. A., and Pendergast, D. D. (1984). Microscopic binding constants in cyclodextrin systems:complexation of $\alpha$-cyclodextrin with sym-1,4-disubstituted benzenes. J. Am. Chem. Soc. 106, 7607-7614. doi: $10.1021 / \mathrm{ja} 00336 \mathrm{a} 048$

Cravotto, G., Di Carlo, S., Tumiatti, V., Roggero, C., and Bremner, H. D. (2005). Degradation of persistent organic pollutants by Fenton's reagent facilitated by microwave or high-intensity ultrasound. Environ. Technol. 26, 721-724. doi: $10.1080 / 09593332608618513$
Cromwell, B., Dubnicka, M., Dubrawski, S., and Levine, M. (2019). Identification of 15 phthalate esters in commercial cheese powder via cyclodextrin-promoted fluorescence detection. ACS Omega 4, 17009-17015. doi: 10.1021/acsomega.9b02585

den Braver-Sewradj, S. P., van Spronsen, R., and Hessel, E. V. S. (2020). Substitution of bisphenol A: a review of the carcinogenicity, reproductive toxicity, and endocrine disruption potential of alternative substances. Crit Rev. Toxicol. 50, 128-147. doi: 10.1080/10408444.2019.1701986

DiScenza, D. J., Lynch, J., Feder, E., and Levine, M. (2018). Detection of bisphenol $\mathrm{A}$ and derivatives in human urine via cyclodextrin-promoted fluorescence modulation. Anal. Methods 10, 3783-3790. doi: 10.1039/C8AY00733K

Dodd, M. C. (2012). Potential impacts of disinfection processes on elimination and deactivation of antibiotic resistance genes during water and wastewater treatment. J. Environ. Monitor. 14, 1754-1771. doi: 10.1039/c2em00006g

Eberle, D., Ball, R., and Boving, T. B. (2017). Impact of ISCO treatment on PFAA Co-contaminants at a former fire training area. Environ. Sci. Technol. 51, 5127-5136. doi: 10.1021/acs.est.6b06591

Foo, K. Y., and Hameed, B. H. (2010). Decontamination of textile wastewater via $\mathrm{TiO}_{2}$ /activated carbon composite materials. Adv. Colloid Interface Sci. 159, 130-143. doi: 10.1016/j.cis.2010.06.002

Hamai, S. (2010). Complex formation of tetrakis(4-sulfonatophenyl)porphyrin with $\gamma$-cyclodextrin, phenylalanine, and tryptophan in aqueous solution. J. Inclusion Phenom. Macrocyclic Chem. 67, 471-481. doi: 10.1007/s10847-009-9730-7

Haynes, A., Halpert, P., and Levine, M. (2019). Colorimetric detection of aliphatic alcohols in $\beta$-cyclodextrin solutions. ACS Omega 4, 18361-18369. doi: 10.1021 /acsomega.9b02612

He, S., Ruan, B., Zheng, Y., Zhou, X., and Xu, X. (2014). Immobilization of chlorine dioxide modified cells for uranium absorption. J. Environ. Radioact. 137, 46-51. doi: 10.1016/j.jenvrad.2014.06.016

Hsu, C.-S., and Huang, D.-J. (2015). Disinfection of herbal spa pool using combined chlorine dioxide and sodium hypochlorite treatment. Environ. Monitoring Assess. 187, 1-11. doi: 10.1007/s10661-014-4242-3

Joseph, C. G., Li, P. G., Bono, A., and Krishnaiah, D. (2009). Sonophotocatalysis in advanced oxidation process: a short review. Ultrasonics Sonochem. 16, 583-589. doi: 10.1016/j.ultsonch.2009.02.002

Kim, D., Amy, G. L., and Karanfil, T. (2015). Disinfection by-product formation during seawater desalination: a review. Water Res. 81, 343-355. doi: 10.1016/j.watres.2015.05.040

Kim, J. G., Yousef, A. E., and Dave, S. (1999). Application of ozone for enhancing the microbiological safety and quality of foods: a review. J. Food Protect. 62, 1071-1087. doi: 10.4315/0362-028X-62.9.1071

Laxma Reddy, P. V., Kavitha, B., Kumar Reddy, P. A., and Kim, K.-H. (2017). $\mathrm{TiO}_{2}$-based photocatalytic disinfection of microbes in aqueous media: a review. Environ. Res. 154, 296-303. doi: 10.1016/j.envres.2017.01.018

Li, X.-F., and Mitch, W. A. (2018). Drinking water disinfection byproducts (DBPs) and human health effects: multidisciplinary challenges and opportunities. Environ. Sci. Technol. 52, 1681-1689. doi: 10.1021/acs.est.7b05440

Loginova, I. V., Rodygin, K. S., Rubtsova, S. A., Slepukhin, P. A., Kuchin, A. V., and Polukeev, V. A. (2011). Oxidation of polyfunctional sulfides with chlorine dioxide. Russian J. Org. Chem. 47, 124-130. doi: 10.1134/S1070428011010167

Lopez, O. L., Marinescu, L., and Bols, M. (2007). New cup-shaped $\alpha$-cyclodextrin derivatives and a study of their catalytic properties in oxidation reactions. Tetrahedron 63, 8872-8880. doi: 10.1016/j.tet.2007.06.018

Ma, J.-W., Huang, B.-S., Hsu, C.-W., Peng, C.-W., Cheng, M.-L., Kao, J.-Y., et al. (2017). Efficacy and safety evaluation of a chlorine dioxide solution. Int. J. Environ. Res. Public Health 14, 329/1-329/12. doi: 10.3390/ijerph14030329

Meireles, A., Giaouris, E., and Simoes, M. (2016). Alternative disinfection methods to chlorine for use in the fresh-cut industry. Food Res. Int. 82, 71-85. doi: 10.1016/j.foodres.2016.01.021

Onundi, Y., Drake, B. A., Malecky, R. T., DeNardo, M. A., Mills, M. R., Kundu, S., et al. (2017). A multidisciplinary investigation of the technical and environmental performances of TAML/peroxide elimination of bisphenol A compounds from water. Green Chem. 19, 4234-4262. doi: 10.1039/C7GC01415E

Oturan, N., Sires, I., Oturan, M. A., and Brillas, E. (2009). Degradation of pesticides in aqueous medium by electro-fenton and related methods. a review. J. Environ. Eng. Manage. 19, 235-255. 
Palcso, B., Moldovan, Z., Suvegh, K., Herczegh, A., and Zelko, R. (2019). Chlorine dioxide-loaded poly(acrylic acid) gels for prolonged antimicrobial effect. Mater. Sci. Eng. C 98, 782-788. doi: 10.1016/j.msec.2019.01.043

Pendergast, D. D., and Connors, K. A. (1985). Complexes of disubstituted benzene positional isomers with $\alpha$-cyclodextrin. Bioorg. Chem. 13, 150-157. doi: 10.1016/0045-2068(85)90017-3

Przybylinska, P. A., and Wyszkowski, M. (2016). Environmental contamination with phthalates and its impact on living organisms. Ecol. Chem. Eng. S 23, 347-356. doi: 10.1515/eces-2016-0024

Roselet, L., and Kumari, J. P. (2017). Inclusion studies on $\alpha$-cyclodextrin complexes of glipizide and gliclazide with effect of pH. Asian J. Pharm. Clin. Res. 10, 273-280. doi: 10.22159/ajpcr.2017.v10i1.15164

Saokham, P., Do, T. T., Van den Mooter, G., and Loftsson, T. (2018). Inclusion complexes of p-hydroxybenzoic acid esters and $\gamma$-cyclodextrin. J. Inclusion Phenom. Macrocyclic Chem. 90, 111-122. doi: 10.1007/s10847-017-0776-7

Schifman, L. A., Kasaraneni, V. K., Sullivan, R. K., Oyanedel-Craver, V., and Boving, T. B. (2016). Bacteria removal from stormwater runoff using tree filters: a comparison of a conventional and an innovative system. Water $8,76 / 1-76 / 16$. doi: $10.3390 /$ w8030076

Serio, N., Miller, K., and Levine, M. (2013). Efficient detection of polycyclic aromatic hydrocarbons and polychlorinated biphenyls via three-component energy transfer. Chem. Commun. 49, 4821-4823. doi: 10.1039/c3cc $40534 \mathrm{f}$

Serio, N., Moyano, D. F., Rotello, V. M., and Levine, M. (2015). Arraybased detection of persistent organic pollutants via cyclodextrin promoted energy transfer. Chem. Commun. 51, 11615-11618. doi: 10.1039/C5CC $04153 \mathrm{H}$

Shah, A., Shahzad, S., Munir, A., Nadagouda, M. N., Khan, G. S., Shams, D. F., et al. (2016). Micelles as soil and water decontamination agents. Chem. Rev. 116, 6042-6074. doi: 10.1021/acs.chemrev.6b 00132

Szente, L., Singhal, A., Domokos, A., and Song, B. (2018). Cyclodextrins: assessing the impact of cavity size, occupancy, and substitutions on cytotoxicity and cholesterol homeostasis. Molecules 23, 1228/1-1228/15. doi: 10.3390/molecules23051228

Wambaugh, J. F., Setzer, R. W., Reif, D. M., Gangwal, S., Mitchell-Blackwood, J., Arnot, J. A., et al. (2013). High-throughput models for exposure-based chemical prioritization in the ExpoCast project. Environ. Sci. Technol. 47, 8479-8488. doi: 10.1021/es400482g

Wang, Y., Liu, H., Liu, G., and Xie, Y. (2014). Oxidation of diclofenac by aqueous chlorine dioxide: identification of major disinfection byproducts and toxicity evaluation. Sci. Total Environ. 473-474, 437-445. doi: 10.1016/j.scitotenv.2013.12.056

Wu, L.-H., Zhang, X.-M., Wang, F., Gao, C.-J., Chen, D., Palumbo, J. R., et al. (2018). Occurrence of bisphenol $S$ in the environment and implications for human exposure: a short review. Sci. Total Environ. 615, 87-98. doi: 10.1016/j.scitotenv.2017.09.194

Xiao, J., Wang, G., Xue, X., Wu, F., Luan, H., and Deng, N. (2007). Enhanced photodegradation behavior of bisphenol $F$ in the presence of $\beta$-cyclodextrin under UV light. Environ. Eng. Sci. 24, 812-820. doi: 10.1089/ees.2006.0107

Yang, Z.-X., Chen, Y., and Liu, Y. (2008). Inclusion complexes of bisphenol A with cyclomaltoheptaose ( $\beta$-cyclodextrin): solubilization and structure. Carbohydr. Res. 343, 2439-2442. doi: 10.1016/j.carres.2008.06.018

Yu, Z., Grasso, M. F., Sorensen, H. H., and Zhang, P. (2019). Ratiometric SERS detection of polycyclic aromatic hydrocarbons assisted by $\beta$ cyclodextrin-modified gold nanoparticles. Microchim. Acta 186:391. doi: 10.1007/s00604-019-3511-9

Conflict of Interest: AB was employed by the company International Dioxcide.

The remaining authors declare that the research was conducted in the absence of any commercial or financial relationships that could be construed as a potential conflict of interest. The authors declare that this study received funding from International Dioxcide. The funder was not involved in the study design, collection, analysis, interpretation of data, the writing of this article, or the decision to submit it for publication.

Copyright (C) 2020 Chaudhuri, DiScenza, Boving, Burke and Levine. This is an openaccess article distributed under the terms of the Creative Commons Attribution License (CC BY). The use, distribution or reproduction in other forums is permitted, provided the original author(s) and the copyright owner(s) are credited and that the original publication in this journal is cited, in accordance with accepted academic practice. No use, distribution or reproduction is permitted which does not comply with these terms. 